\title{
Direct Evaluation of Hypersingular Galerkin Surface Integrals. II
}

\author{
L. J. Gray \\ Computer Science and Mathematics Division \\ Oak Ridge National Laboratory \\ Oak Ridge, TN 37831-6367 \\ A. Salvadori \\ Department of Civil Engineering \\ University of Brescia \\ via Branze 38 \\ 25123 Brescia, Italy \\ A.-V. Phan \\ Department of Mechanical Engineering \\ University of South Alabama \\ Mobile, AL 36688 \\ V. Mantič \\ Grupo de Elasticidad y Resistencia de Materiales \\ University of Sevilla \\ Camino de los Descubrimientos, s/n \\ 41092 Sevilla, Spain
}

\begin{abstract}
Direct boundary limit algorithms for evaluating hypersingular Galerkin surface integrals have been successful in identifying and removing the divergent terms, leaving finite integrals to be evaluated. This paper is concerned with the numerical computation of these multi-dimensional integrals. The integrands contain a weakly singular logarithmic term that is difficult to evaluate directly using standard numerical techniques. Herein it is shown that analytic integration of these weakly singular terms can be accomlished by suitably re-ordering the parameter integrals. In addition to improved accuracy, this process also reduces the dimension of the numerical quadrature, and hence improves efficiency.
\end{abstract}

\section{Introduction}

This paper is a continuation of the work in [14] on the direct evaluation of Galerkin hypersingular boundary integrals in three dimensions. In a Galerkin formulation $[3,5]$, the boundary integral equation involves a double integration over the boundary, and in a numerical approximation, the individual integrals to be computed 
take the form

$$
\int_{E_{P}} \psi_{k}(P) \int_{E_{Q}} \psi_{j}(Q) \mathcal{K}(P, Q) \mathrm{d} Q \mathrm{~d} P
$$

The kernel function $\mathcal{K}(P, Q)$ can be the Green's function $G(P, Q)$ for the problem or one of its derivatives, but herein, as in [14], the focus is on the hypersingular second derivative. The integrations are over two elements, $E_{P}$ and $E_{Q}$, that comprise part of the approximation of the boundary surface, and the functions $\psi_{j}(Q)$ in Eq. (1) are the chosen shape functions that define the approximations on the boundary (e.g., linear, quadratic). These functions are then normally used as the Galerkin weight functions $\psi_{k}(P)$ for the outer integration.

The term direct applied to the evaluation methods presented in [14] has two meanings. First, in contrast to the most commonly employed technique based upon Stokes' Theorem [9, 19], there is no reformulation of the integrand in Eq. (1). Second, the individual integrals when $E_{P}=E_{Q}$ (coincident) or when $E_{P}$ and $E_{Q}$ share an edge (edge-adjacent) are divergent, and a direct method will explicitly compute the divergent terms. This is in contrast to methods based upon the well known Hadamard Finite Part definition [18, 20], for which the divergences disappear through the application of this definition [1, 2, 21, 22, 8, 23]. In either approach, numerical evaluation has been successfully carried out using a Duffy transformation [12] to help mollify the singularities; herein, analytic treatment of the singularties will leave, for the numerical work, integrals that can be easily handled by standard Gauss quadrature.

Herein, as in [14], the integrals are defined and evaluated as a boundary limit

$$
\lim _{\epsilon \rightarrow 0} \int_{E_{P}} \psi_{k}(P) \int_{E_{Q}} \psi_{j}(Q) \mathcal{K}\left(P_{\epsilon}, Q\right) \mathrm{d} Q \mathrm{~d} P
$$

where $P_{\epsilon}$ denotes points exterior to the volume that converge to the boundary point $P \in E_{P}$ as $\epsilon$ tends to zero. For two dimensional analysis, an alternative direct formulation method (employing a different limit process) has been presented in [4] (see also [10,17]); in a collocation approximation, this method has also been shown to be successful in handling singularities beyond hypersingular [11]. Similarly, [16] presents an efficient and accurate direct Galerkin method for post-processing the surface gradient.

The previous work [14] established main two results. First, as noted above, the individual coincident and edge-adjacent edge hypersingular integrals are separately divergent: if the boundary limit is accomplished by setting $P_{\epsilon}=P+\epsilon \mathbf{N}$, $\mathbf{N}=\mathbf{N}(P)$ the unit outward normal at $P$, then for $\epsilon \rightarrow 0$, the edge and coincident integrals behave as $\log \left(\epsilon^{2}\right)$. Second, the complete boundary integration of the hypersingular kernel is finite, as all divergent terms can be shown to cancel when the total integral is assembled. This analysis was accomplished by evaluating the four-dimensional parameter space integral partially analytically. For the coincident computation, two dimensions were integrated analytically, leaving 
a finite double integral. For the adjacent edge, the remaining finite integral was three-dimensional. The exact integration explicitly indentified the $\log \left(\epsilon^{2}\right)$ term, and at the same time, the remainder of the analytic expression was seen to be finite at $\epsilon=0$ and integrable with respect to the remaining parameters. With the divergent terms identified and removed exactly, the calculation can proceed with well defined finite integrals.

This paper is concerned with the last stage of this singular integration program, the evaluation of the finite integrals that remain after the boundary limit, $\epsilon=0$, has been completed. The difficulty at this point is that the same terms that gave rise to the divergent $\log \left(\epsilon^{2}\right)$ expressions also produce integrands containing an integrable logarithmic singularity. The simplest approach would be to invoke well-known Gauss rules for handling this weakly singular function [6]. However, in the present circumstances, this method would be difficult to apply: the singularity does not appear in a simple form that allows easy implementation of this numerical technique.

It will be shown that this problem can be remedied by integrating the logarithmic singularity analytically. However, this exact integration cannot be immediately implemented, for the same reason that use of the special Gauss rule is difficult: the logarithmic singularity stems from the last parameter to be integrated in the multi-dimensional parameter integrals. The key step is therefore to re-order the integrations, and this process is described herein. Once the re-ordering is accomplished, numerical methods could be invoked; however at this point the innermost integral is simple enough to be computed analytically. There is nothing especially difficult in the manipulation of the integrals - it is simply elementary calculus. However, this re-ordering is key: a standard Gauss quadrature evaluation of the finite integrals in their original form performs quite poorly. As shown below, this calculation in general fails to converge even at 24 Gauss points, whereas the analytic approach has converged at 8 points.

Although the most important objective must be to obtain a reliable evaluation of the hypersingular integrals, the new algorithms also improve efficiency. Instead of two and three dimensional integrals, the numerical quadrature is reduced to one and two dimensions, and, as just noted, far fewer Gauss points are required. Thus, these methods can also be profitably applied to for the less singular kernel functions, the Green's function and its first derivative, even though the problematic logarithm function does not appear.

As in [14], the algorithms will be presented in the context of a linear element. We wish to emphasize, however, that the techniques are not limited to this situation. For higher order interpolation, the basic idea is to split the integrand into a nonsingular part that can be safely evaluated numerically, plus a singular component that is subject to analytic integration and limit evaluation. This singular term is constructed so as to have the same form as in the linear interpolation, and this 
is the reason that it suffices to examine the linear case. For two dimensions, the splitting into singular and nonsingular components has been described in [13], and the procedures in three dimensions are essentially the same.

The following section provides a brief summary of the methods developed in [14], sufficient for a discussion of the new follow-on algorithms. More complete details can of course be found in this reference. The subsequent two sections then discuss the treatment of the coincident and adjacent edge hypersingular integrations, respectively. The effectiveness of these algorithms is established by results from test calculations presented in section 5 .

\section{Boundary Limit Evaluation}

As just noted, the purpose of this section is to review, as briefly as possible, the formulation in [14]. The integration methods are applicable to all singular integrals commonly employed in boundary integral analysis, namely $G$ and its first and second derivatives, incorporating most, if not all, Green's functions. However, for purposes of discussion, it is convenient to employ the terminology and notation of the Laplace equation, $\nabla^{2} \phi=0$. The Green's function is the point source potential

$$
G(P, Q)=\frac{1}{4 \pi r}=\frac{1}{4 \pi} \frac{1}{\|Q-P\|},
$$

and thus the hypersingular kernel is

$$
\mathcal{K}(P, Q)=\frac{\partial^{2} G}{\partial \mathbf{N} \partial \mathbf{n}}(P, Q)=\frac{1}{4 \pi}\left(\frac{\mathbf{n} \cdot \mathbf{N}}{r^{3}}-3 \frac{(\mathbf{n} \cdot \mathbf{R})(\mathbf{N} \cdot \mathbf{R})}{r^{5}}\right) .
$$

Here $\mathbf{R}=Q-P, r=\|Q-P\|$ and $\mathbf{n}=\mathbf{n}(Q), \mathbf{N}=\mathbf{N}(P)$ is the unit outward normal on the boundary. The integrals involving $G$ and its first order derivative lead to simpler integrals that can be handled by the same methods.

As noted in [14], it suffices to consider a linear element, as these techniques form the basis for treating higher order interpolations. We choose to work with a 3noded triangular element, the approximations to the boundary and the boundary functions are defined using an equilateral triangle parameter space $\{\eta, \xi\},-1 \leq$ $\eta \leq 1,0 \leq \xi \leq \sqrt{3}(1-|\eta|)$. The three linear shape functions $\psi_{j}(\eta, \xi)$ are given by

$$
\begin{aligned}
& \psi_{1}(\eta, \xi)=\frac{\sqrt{3}(1-\eta)-\xi}{2 \sqrt{3}} \\
& \psi_{2}(\eta, \xi)=\frac{\sqrt{3}(1+\eta)-\xi}{2 \sqrt{3}} \\
& \psi_{3}(\eta, \xi)=\frac{\xi}{\sqrt{3}} .
\end{aligned}
$$


The parametric variables for the outer $P$ integration will be denoted by $(\eta, \xi)$, and that for $Q$ by $\left(\eta^{*}, \xi^{*}\right)$. Thus, for an element defined by nodal points $\left\{Q_{j}=\left(x_{j}, y_{j}, z_{j}\right)\right\}$, the mapping from parameter space to the approximate boundary surface is

$$
Q\left(\eta^{*}, \xi^{*}\right)=\sum_{j=1}^{3}\left(x_{j}, y_{j}, z_{j}\right) \psi_{j}\left(\eta^{*}, \xi^{*}\right) .
$$

In Eq. (1) $\psi_{j}\left(\eta^{*}, \xi^{*}\right)$ appears due to the representation of the coefficient function (in this case, the surface potential $\phi(Q)$ ) in terms of nodal values and the shape functions. The function $\psi_{k}(\eta, \xi)$ is the weight function in the Galerkin formulation of the integral equation.

\subsection{Coincident integral}

For $E_{P}=E_{Q}=E$, the coincident integral to be evaluated is

$$
J_{P}^{2} \int_{-1}^{1} \int_{0}^{\boldsymbol{\xi}(\eta)} \psi_{k}(\eta, \xi) \mathrm{d} \xi \mathrm{d} \eta \int_{-1}^{1} \int_{0}^{\boldsymbol{\xi}\left(\eta^{*}\right)} \psi_{j}\left(\eta^{*}, \xi^{*}\right) \frac{\partial^{2} G}{\partial \mathbf{N} \partial \mathbf{n}}(P, Q) \mathrm{d} \xi^{*} \mathrm{~d} \eta^{*}
$$

where $E$ is defined by nodes $P_{k}, 1 \leq k \leq 3, J_{P}\left(=J_{Q}\right)$ is the (constant) jacobian for the element $E$ and $\boldsymbol{\xi}(\eta)=\sqrt{3}(1-|\eta|)$. For the inner $Q$ integration, the first step is to define a polar coordinate system centered at $P=(\eta, \xi)$,

$$
\begin{aligned}
& \eta^{*}-\eta=\rho \cos (\theta) \\
& \xi^{*}-\xi=\rho \sin (\theta)
\end{aligned}
$$

as illustrated in Fig. 1.

The expression for the upper limit of $\rho, 0<\rho<\rho_{L}(\theta)$, is different as $\theta$ traverses each edge, and thus the $(\rho, \theta)$ integration must be split into three subtriangles. Again, as discussed in [14], it suffices to carry out the calculation for the lower subtriangle associated with the edge $\xi^{*}=0$. In this case, the integration limits are $\rho_{L}=-\xi / \sin (\theta)$ and $\Theta_{1} \leq \theta \leq \Theta_{2}$ where

$$
\begin{aligned}
\Theta_{1} & =-\frac{\pi}{2}-\tan ^{-1}\left(\frac{1+\eta}{\xi}\right) \\
\Theta_{2} & =-\frac{\pi}{2}+\tan ^{-1}\left(\frac{1-\eta}{\xi}\right) .
\end{aligned}
$$

With $P$ replaced by $P+\epsilon \mathbf{N}$ for the exterior limit, the distance function takes the simple form

$$
r^{2}(\rho, \theta)=\epsilon^{2}+a^{2}(\theta) \rho^{2},
$$

where

$$
a^{2}(\theta)=a_{c c} \cos ^{2}(\theta)+a_{c s} \cos (\theta) \sin (\theta)+a_{s s} \sin ^{2}(\theta) .
$$




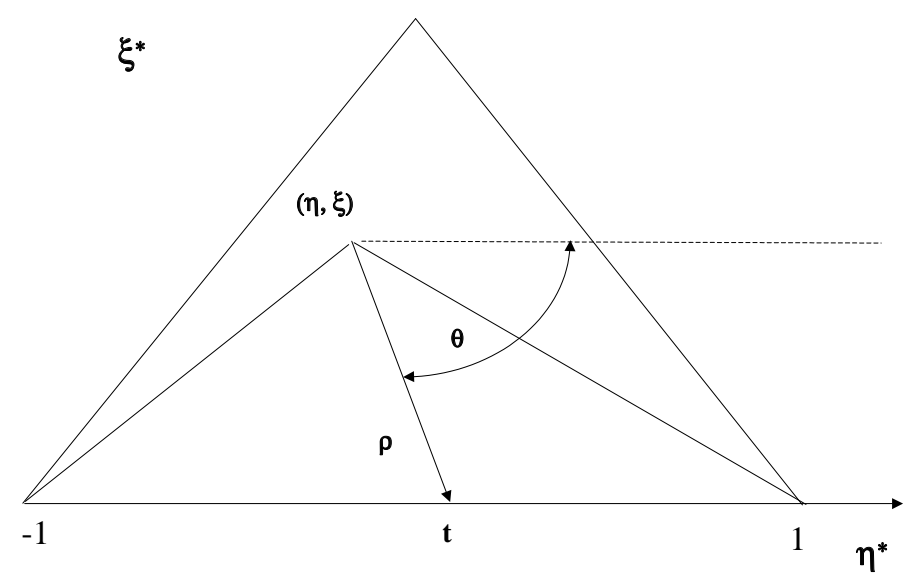

Figure 1: First polar coordinate transformation, $\left\{\eta^{*}, \xi^{*}\right\} \rightarrow\{\rho, \theta\}$, for the coincident integration. The variable $t$ eventually replaces $\theta$.

The three coefficients $a_{\alpha \beta}, \alpha, \beta=c, s$ depend solely on the coordinates of the element nodes. With Eq. (8), the shape functions $\psi_{j}(Q)$ are linear functions of $\rho$, and thus the integral with respect to $\rho$ from 0 to $\rho_{L}(\theta)$ is easily evaluated. After this integration, the most singular integral becomes

$$
-\frac{1}{4 \pi} \int_{-1}^{1} \mathrm{~d} \eta \int_{0}^{\boldsymbol{\xi}(\eta)} \psi_{k}(\eta, \xi) \mathrm{d} \xi \int_{\Theta_{1}}^{\Theta_{2}} \frac{\rho_{L}^{2}}{\left(\epsilon^{2}+a^{2} \rho_{L}^{2}\right)^{3 / 2}} \mathrm{~d} \theta,
$$

and as $\rho_{L}$ can become small for $\xi \approx 0$, this expression is still singular at $\epsilon=0$. As the dependence of the integrand on $\theta$ is harmless, the $\xi$ and $\theta$ integrations are interchanged by introducing the variable $t,-1 \leq t \leq 1$,

$$
\theta=-\frac{\pi}{2}+\tan ^{-1}\left(\frac{t-\eta}{\xi}\right)
$$

in which case $\rho_{L}=\left(\xi^{2}+(t-\eta)^{2}\right)^{1 / 2}$. As indicated in Figure 1, $t$ is the 'end-point' $(t, 0)$ of $\rho$ on the $\eta^{*}$-axis, and the singularity is at $t=\eta, \xi=0$. Moving the $\xi$ integration forward in Eq. (12), and introducing a second polar coordinate system $\{\Lambda, \Psi\}$

$$
\begin{aligned}
t & =\Lambda \cos (\Psi)+\eta \\
\xi & =\Lambda \sin (\Psi)
\end{aligned}
$$


to replace $\{t, \xi\}$, Eq. (12) results in integrals of the form

$$
\int_{-1}^{1} \mathrm{~d} \eta \int_{\Psi_{l}}^{\Psi_{l+1}} \sin (\Psi) \mathrm{d} \Psi \int_{0}^{\Lambda_{L}} \Lambda^{s} \frac{\Lambda^{2}}{\left(\epsilon^{2}+a^{2} \Lambda^{2}\right)^{3 / 2}} \mathrm{~d} \Lambda .
$$

where

$$
\Lambda_{L}=\sqrt{3}(1+\eta) / \sin (\Psi) .
$$

The powers of $\Lambda, s=0,1,2$, stem from the product of the $Q$ and $P$ shape functions, and the $\sin (\Psi)$ from the change of variables. The $\Lambda$ integral can be evaluated analytically, with the $s=0$ integral producing the terms of interest: a $\log \left(\epsilon^{2}\right)$ divergent term, plus a term of the form $\log \left(a^{2} \Lambda_{L}^{2}\right)$. Note that the $\{t, \xi\}$ domain, Fig. 2, is a rectangle, and integrating over $\{\Lambda, \Psi\}$ will necessitate a decomposition into three subdomains $\Psi_{l} \leq \Psi \leq \Psi_{l+1}$, where $\Psi_{0}=0, \Psi_{3}=\pi$ and

$$
\Psi_{1}=\tan ^{-1}(\boldsymbol{\xi}(\eta) /(1-\eta)) \quad \Psi_{2}=\pi-\tan ^{-1}(\boldsymbol{\xi}(\eta) /(1+\eta))
$$

Most importantly $\Lambda_{L} \rightarrow 0$ for $\eta \rightarrow \pm 1$, and it is the presence of the weakly

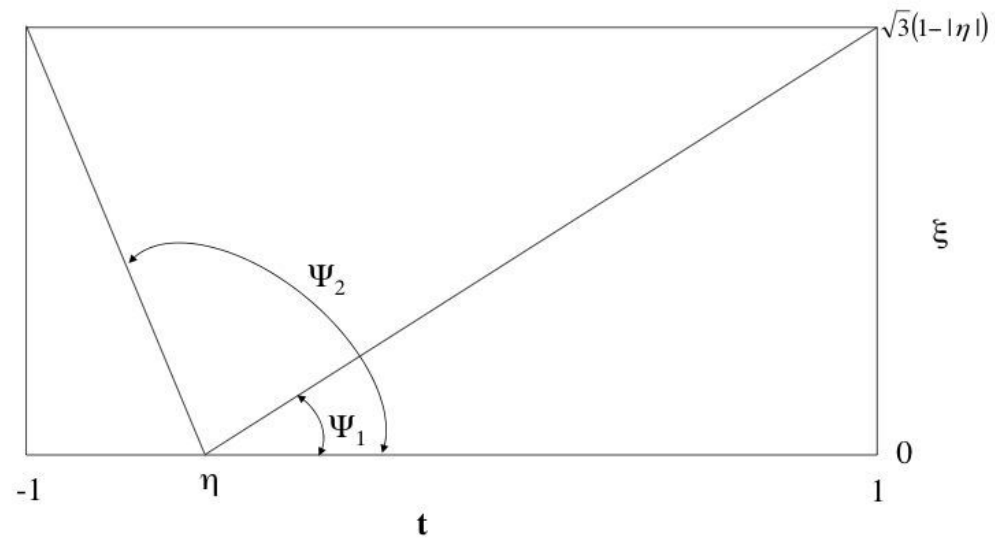

Figure 2: Geometry of the second polar coordinate transformation, $\{t, \xi\} \rightarrow$ $\{\Lambda, \Psi\}$, for the coincident integration.

singular $\log \left(a^{2} \Lambda_{L}^{2}\right)$ term that complicates the numerical evaluation.

It is tempting, as this would be the simplest approach, to try to evaluate this logarithm integral by employing the effective numerical methods [6] designed to 
handle an integral of the form

$$
\int_{0}^{1} f(x) \log (x) \mathrm{d} x
$$

However, as noted in the Introduction, these methods would be somewhat awkward to apply here. In the first place, $\Lambda_{L}=\Lambda_{L}(\eta, \Psi)$ and while the vanishing of $\Lambda_{L}$ is primarily a function of $\eta \rightarrow \pm 1$, it also depends upon $\Psi$. For discussion purposes consider $\eta \approx-1$, and note that the width of the rectangle in Fig. 2 approaches zero. Also, $\Psi_{1} \rightarrow 0, \Psi_{2} \rightarrow 2 \pi / 3$, and in the middle triangle in Fig. $2, \Psi_{1}<\Psi<\Psi_{2}$. To apply the numerical integration, it is necessary to split the logarithm as

$$
\log \left(a^{2} \Lambda_{L}^{2}\right)=\log \left(\frac{3 a^{2}}{\sin ^{2}(\Psi)}\right)+2 \log (1+\eta)
$$

to isolate the singularity at $\eta=-1$ in the appropriate form of Eq. (18). However, this requires yet another splitting of the integral, as it would be necessary to avoid the situation $\sin (\Psi) \approx 0$. Thus, repeating the comment above, the logarithmic singularity is a function of both $\eta$ and $\Psi$, and attempts to separate the two parameters in the definition of $\Lambda_{L}$ appears to create additional problems.

Second, there is more to the integral over $\{\eta, \Psi\}$ than the logarithm term, and thus this component would be handled differently than the rest of the integral. In the algorithm presented below, the $\{\eta, \Psi\}$ integral is treated as a whole, the singularity is handled analytically, and the numerical integration is one dimensional instead of two. This will however require a further decomposition into cases, but on the whole appears to be simpler and to offer significant advantages over a completely numerical treatment.

\subsection{Edge Adjacent Integration}

As the edge adjacent integral produces the counterbalancing $\log \left(\epsilon^{2}\right)$ divergent term, it is also to be expected that it produces a finite logarithm integral as well. To begin, orient the elements so that the shared edge is defined by $\xi=0$ in $E_{P}$, and $\xi^{*}=0$ for $E_{Q}$, and the singularity $P=Q$ is therefore characterized by $\eta+\eta^{*}=\xi=\xi^{*}=0$. Employ polar coordinates for the $Q$ integration,

$$
\begin{aligned}
& \eta^{*}=\rho \cos (\theta)-\eta \\
& \xi^{*}=\rho \sin (\theta)
\end{aligned}
$$

and as shown in Fig. 3(a), the $\theta$ integration must be split into two pieces

$$
\int_{-1}^{1} \mathrm{~d} \eta \int_{0}^{\sqrt{3}(1-|\eta|)} \mathrm{d} \xi\left[\int_{0}^{\Theta_{1}(\eta)} \mathrm{d} \theta \int_{0}^{L_{1}^{+}} \rho \mathrm{d} \rho+\int_{\Theta_{1}(\eta)}^{\pi} \mathrm{d} \theta \int_{0}^{L_{1}^{-}} \rho \mathrm{d} \rho\right],
$$


where $L_{1}^{ \pm}=\sqrt{3}(1 \pm \eta) /(\sin (\theta) \pm \sqrt{3} \cos (\theta))$. For simplicity the integrands have been omitted, but it will be useful to retain the jacobians of the various transformations.

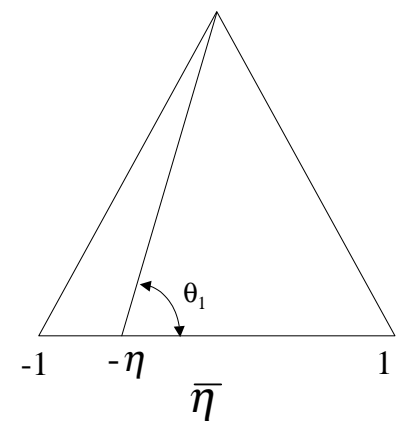

(a)

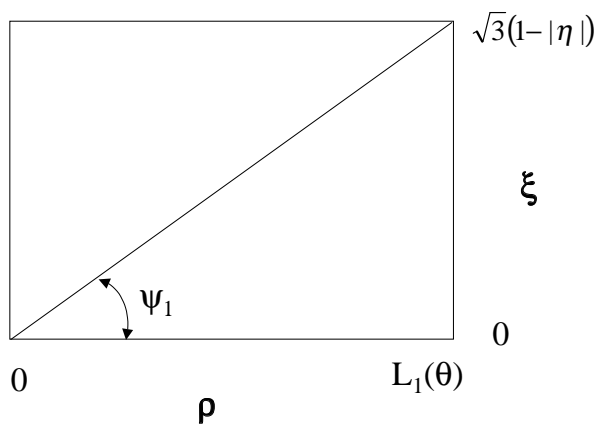

(b)

Figure 3: (a) Polar coordinate transformation employed in the $Q$ element, $\left\{\eta^{*}, \xi^{*}\right\} \rightarrow\{\rho, \theta\} ;($ b) Second polar coordinate transformation $\{\rho, \xi\} \rightarrow\{\Lambda, \Psi\}$ for the edge-adjacent integration.

The key observation is that the break-point in $\theta$,

$$
\theta_{1}(\eta)=\frac{\pi}{2}-\tan ^{-1}\left(\frac{\eta}{\sqrt{3}}\right)
$$

is only a function of $\eta$. the integrations can therefore be rearranged,

$$
\begin{aligned}
& \int_{-1}^{1} \mathrm{~d} \eta \int_{0}^{\Theta_{1}(\eta)} \mathrm{d} \theta \int_{0}^{\sqrt{3}(1-|\eta|)} \mathrm{d} \xi \int_{0}^{L_{1}^{+}} \rho \mathrm{d} \rho+ \\
& \int_{-1}^{1} \mathrm{~d} \eta \int_{\Theta_{1}(\eta)}^{\pi} \mathrm{d} \theta \int_{0}^{\sqrt{3}(1-|\eta|)} \mathrm{d} \xi \int_{0}^{L_{1}^{-}} \rho \mathrm{d} \rho .
\end{aligned}
$$

As the singularity occurs when $\rho=\xi=0$, it makes sense to now introduce a second polar coordinate transformation

$$
\begin{aligned}
& \rho=\Lambda \cos (\Psi) \\
& \xi=\Lambda \sin (\Psi),
\end{aligned}
$$


as shown in Fig. 3(b). Note that $\Lambda=0$ encapsulates all three conditions for $r=0$, namely $\xi=\xi^{*}=0, \eta=-\eta^{*}$, and thus the analytic integration over $\Lambda$ will suffice to produce the $\log \left(\epsilon^{2}\right)$ term [14]. It also produces a finite integral of the form

$$
\int_{-1}^{1} \mathrm{~d} \eta \int_{\Theta_{l}}^{\Theta_{l+1}} \mathrm{~d} \theta \int_{\Psi_{m}}^{\Psi_{m+1}} \mathcal{A}(\eta, \theta, \Psi) \mathrm{d} \Psi
$$

where $l, m=0,1$, and $\Theta_{0}=0, \Theta_{2}=\pi, \Psi_{0}=0, \Psi_{2}=\pi / 2$. The problem is that once again $\mathcal{A}$ contains a logarithm function that is weakly singular for

$\eta \rightarrow \pm 1$, (respectively) $L_{1}^{\mp} \rightarrow 0$ (Fig. 3 ). As the angle integrals cannot be executed analytically, it will therefore be necessary to arrange for the $\eta$ integration to be innermost of the three.

\section{Re-ordering: Coincident}

Recall that the coincident integral has been reduced to an integration over $\{\eta, \Psi\}$, with the $\Psi$ integral decomposed into three subintegrals and the weak singularity at $\eta= \pm 1$. The goal is to re-order the integrals and integrate $\eta$ analytically, and thus each subintegral has to be examined separately. It is also necessary to treat the weak singularities $\eta=1$ and $\eta=-1$ separately, which is conveniently accomplished by splitting the $\eta$ integral as $-1<\eta<0$ and $0<\eta<1$.

Our goal here is to describe the re-ordering procedures, and thus in what follows we omit the integrands and the details of the simple integration with respect to $\eta$.

\section{1 $\eta \geq 0$}

In this case, the subdivision of the $\Psi$ integral and the limits of the $\Lambda$ integration are

$$
\begin{array}{ll}
0 \leq \Psi \leq \frac{\pi}{3} & \Lambda_{L}=(1-\eta) / \cos (\Psi) \\
\frac{\pi}{3} \leq \Psi \leq \Psi_{\eta} & \Lambda_{L}=\sqrt{3}(1-\eta) / \sin (\Psi) \\
\Psi_{\eta} \leq \Psi \leq \pi & \Lambda_{L}=-(1+\eta) / \cos (\Psi)
\end{array}
$$

where

$$
\Psi_{\eta} \equiv \Psi_{2}=\pi-\tan ^{-1}(\sqrt{3}(1-\eta) /(1+\eta)) .
$$

The domain of integration is shown in Fig. 4. Interchanging the order of integration, and subsequently evaluating the $\eta$ integral analytically, is a relatively simple process. The three $\Psi$ integrals become

$$
\int_{0}^{1} \mathrm{~d} \eta \int_{0}^{\pi / 3} \mathrm{~d} \Psi=\int_{0}^{\pi / 3} \mathrm{~d} \Psi \int_{0}^{1} \mathrm{~d} \eta=\int_{0}^{\pi / 3} \Phi_{k, j}^{a} \mathrm{~d} \Psi
$$




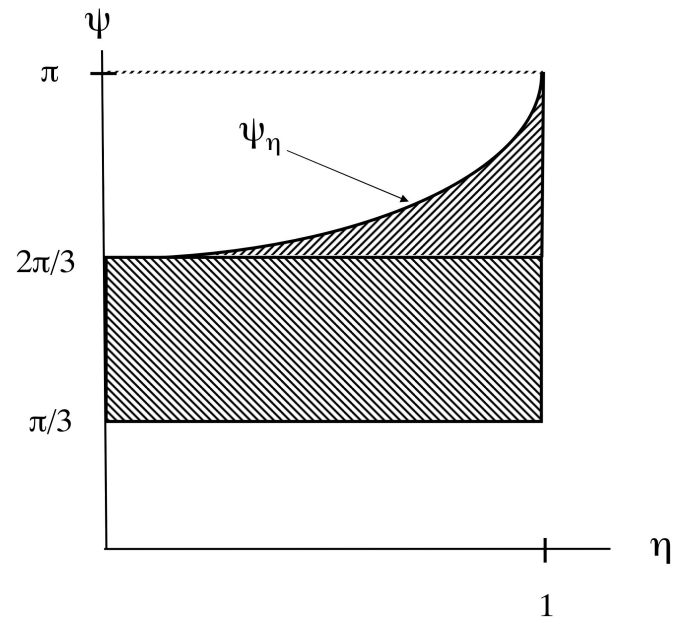

Figure 4: The $\{\eta, \Psi\}$ domain for $\eta>0$.

$$
\begin{aligned}
\int_{0}^{1} \mathrm{~d} \eta \int_{\pi / 3}^{\Psi_{2}} \mathrm{~d} \Psi & =\int_{\pi / 3}^{2 \pi / 3} \mathrm{~d} \Psi \int_{0}^{1} \mathrm{~d} \eta+\int_{2 \pi / 3}^{\pi} \mathrm{d} \Psi \int_{\alpha}^{1} \mathrm{~d} \eta \\
& =\int_{\pi / 3}^{2 \pi / 3} \Phi_{k, j}^{b} \mathrm{~d} \Psi+\int_{2 \pi / 3}^{\pi} \Phi_{k, j}^{c} \mathrm{~d} \Psi \\
\int_{0}^{1} \mathrm{~d} \eta \int_{\Psi_{2}}^{\pi} \mathrm{d} \Psi & =\int_{2 \pi / 3}^{\pi} \mathrm{d} \Psi \int_{0}^{\alpha} \mathrm{d} \eta=\int_{2 \pi / 3}^{\pi} \Phi_{k, j}^{d} \mathrm{~d} \Psi
\end{aligned}
$$

where $\alpha$ is obtained by inverting the formula for $\Psi_{\eta}$,

$$
\alpha=\frac{1-\beta}{1+\beta} \quad \beta=\tan (\pi-\Psi) / \sqrt{3}
$$

The $\Phi_{k, j}^{a-d}$ functions, the result of the $\eta$ analytic integration, are integrated numerically with respect to $\Psi$. 


\section{$3.2 \eta \leq 0$}

Not surprisingly, the procedures for $\eta \leq 0$ are essentially the same as above. The subdivision of the $\Psi$ integral is

$$
\begin{array}{ll}
0 \leq \Psi \leq \Psi_{\eta} & \Lambda_{L}=(1-\eta) / \cos (\Psi) \\
\Psi_{\eta} \leq \Psi \leq 2 \pi / 3 & \Lambda_{L}=\sqrt{3}(1-\eta) / \sin (\Psi) \\
2 \pi / 3 \leq \Psi \leq \pi & \Lambda_{L}=-(1+\eta) / \cos (\Psi)
\end{array}
$$

where now $\Psi_{\eta}=\tan ^{-1}(\sqrt{3}(1+\eta) /(1-\eta))$, and the new $\{\eta, \Psi\}$ domain is shown in Fig. 5. Interchanging the order of integration and integrating $\eta$ analytically as before,

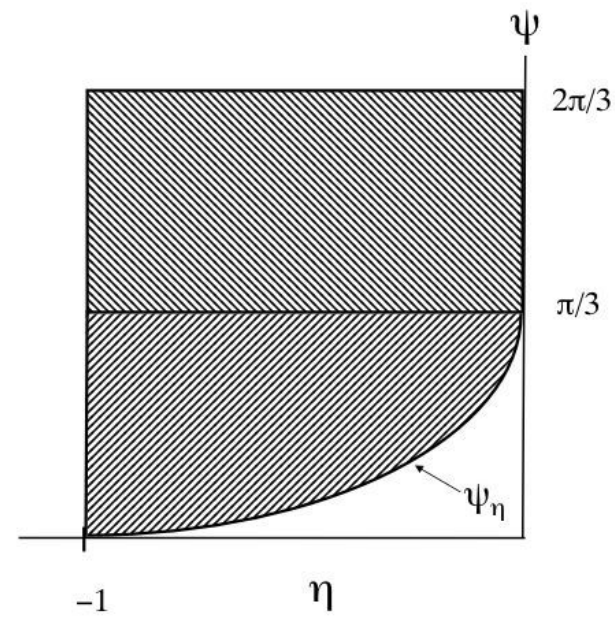

Figure 5: The $\{\eta, \Psi\}$ domain for $\eta<0$.

$$
\begin{aligned}
\int_{-1}^{0} \mathrm{~d} \eta \int_{0}^{\Psi_{\eta}} \mathrm{d} \Psi & =\int_{0}^{\pi / 3} \mathrm{~d} \Psi \int_{\alpha}^{0} \mathrm{~d} \eta=\int_{0}^{\pi / 3} \Phi_{k, j}^{e} \mathrm{~d} \Psi \\
\int_{-1}^{0} \mathrm{~d} \eta \int_{\Psi_{\eta}}^{2 \pi / 3} \mathrm{~d} \Psi & =\int_{0}^{\pi / 3} \mathrm{~d} \Psi \int_{-1}^{\alpha} \mathrm{d} \eta+\int_{\pi / 3}^{2 \pi / 3} \mathrm{~d} \Psi \int_{-1}^{0} \mathrm{~d} \eta \\
& =\int_{0}^{2 \pi / 3} \Phi^{f} \mathrm{~d} \Psi+\int_{\pi / 3}^{2 \pi / 3} \Phi_{k, j}^{g} \mathrm{~d} \Psi
\end{aligned}
$$




$$
\int_{-1}^{0} \mathrm{~d} \eta \int_{2 \pi / 3}^{\pi} \mathrm{d} \Psi=\int_{2 \pi / 3}^{\pi} \mathrm{d} \Psi \int_{-1}^{0} \mathrm{~d} \eta=\int_{2 \pi / 3}^{\pi} \Phi_{k, j}^{h} \mathrm{~d} \Psi
$$

where now

$$
\alpha=-\frac{1-\beta}{1+\beta} \quad \beta=\tan (\Psi) / \sqrt{3}
$$

\section{Re-ordering: Adjacent Edge}

As shown in [14] and indicated by Eq. (25), isolating the divergent term in the adjacent edge integral only requires a single analytic integration. The subsequent integral over $\eta$ and the angles $\theta$ and $\Psi$ from the polar coordinate transformations is finite. However, to treat the weak logarithmic singularity, we once again wish to integrate $\eta$ analytically, and this will require two interchanges. As with the coincident integral, the singularity is at $\eta= \pm 1$, and it is convenient to consider $\eta$ positive and negative separately. In the following we examine $\eta \geq 0$, the remaining case is similar. Moreover, in the implementation of this method, $\eta \geq 0$ suffices: $\eta \leq 0$ can be computed by 'flipping' the elements around and using the $\eta \geq 0$ formulas; this is analogous to the rotation procedure employed for the coincident integration.

The further complication in the edge adjacent case is that, as shown in Fig. 3, both the $\theta$ and $\Psi$ integrals are in two pieces. In particular, the splitting in $\theta$ is $0 \leq \theta \leq \theta_{\eta}$ and $\theta_{\eta} \leq \theta \leq \pi$, and $\theta_{\eta}$ is defined in Eq. (22).

\section{1 $\theta_{\eta} \leq \theta \leq \pi$}

For this subtriangle, the splitting of $\Psi$ integral is independent of $\eta$, and thus this is the simpler of the two cases. The two integrals are

$$
\int_{0}^{1} \mathrm{~d} \eta \int_{\theta_{\eta}}^{\pi} \mathrm{d} \theta \int_{0}^{\Psi_{1}} \mathrm{~d} \Psi+\int_{0}^{1} \mathrm{~d} \eta \int_{\theta_{\eta}}^{\pi} \mathrm{d} \theta \int_{\Psi_{1}}^{\pi / 2} \mathrm{~d} \Psi
$$

where the upper limit on $\Lambda, \Lambda_{L}$, is different in the two terms (see Fig. 3),

$$
\Lambda_{L}= \begin{cases}L_{1}(\theta) / \cos (\Psi) & 0<\Psi<\Psi_{1} \\ \boldsymbol{\xi}(\eta) / \sin (\Psi) & \Psi_{1}<\Psi<\pi / 2\end{cases}
$$

As before $\boldsymbol{\xi}(\eta)=\sqrt{3}(1-|\eta|), L_{1}(\theta)$ is the upper limit on $\rho$ given below Eq. (21) (We now drop the superscript \pm ) and the $\Psi$ integral is split at

$$
\Psi_{1}=\Psi_{1}(\theta)=\tan ^{-1}\left(\boldsymbol{\xi}(\eta) / L_{1}\right)=\tan ^{-1}(\sin (\theta)-\sqrt{3} \cos (\theta)) .
$$


The important simplification in this case is that $\Psi_{1}$ is not a function of $\eta$. Thus, once $\eta$ and $\theta$ are interchanged, the $\eta$ can be moved immediately past the $\Psi$ integral.

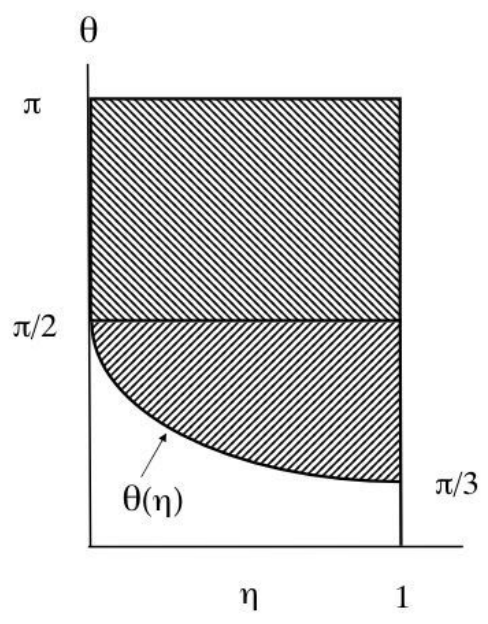

Figure 6: The $\{\eta, \theta\}$ domain for the first shift of the integral, $\theta_{\eta} \leq \theta \leq \pi$.

Noting that $\theta_{\eta}(0)=\pi / 2$ and $\theta_{\eta}(1)=\pi / 3$, the geometry for interchanging $\eta$ and $\theta$ is shown in Fig. 6. Inverting the relationship between $\eta$ and $\theta$ yields

$$
\eta(\theta)=\frac{\sqrt{3}}{\tan (\theta)}
$$

and switching the integrals results in

$$
\begin{aligned}
\int_{\pi / 2}^{\pi} \mathrm{d} \theta \int_{0}^{\Psi_{1}} \mathrm{~d} \Psi \int_{0}^{1} \mathrm{~d} \eta+\int_{\pi / 3}^{\pi / 2} \mathrm{~d} \theta \int_{0}^{\Psi_{1}} \mathrm{~d} \Psi \int_{\eta(\theta)}^{1} \mathrm{~d} \eta+ \\
\int_{\pi / 2}^{\pi} \mathrm{d} \theta \int_{\Psi_{1}}^{\pi / 2} \mathrm{~d} \Psi \int_{0}^{1} \mathrm{~d} \eta+\int_{\pi / 3}^{\pi / 2} \mathrm{~d} \theta \int_{\Psi_{1}}^{\pi / 2} \mathrm{~d} \Psi \int_{\eta(\theta)}^{1} \mathrm{~d} \eta
\end{aligned}
$$

As before, the $\eta$ integral is easily evaluated analytically. 


\section{$4.20<\theta \leq \theta_{\eta}$}

Referring to Fig. 3, we note that as $\eta>0$, the upper limit of $\rho, L_{1}(\theta)$ now does not approach zero. However, the upper limit of $\Lambda$, which appears in the logarithm function, is

$$
\Lambda_{L}=\left\{\begin{array}{ll}
L_{1}(\theta) / \cos (\Psi) & 0<\Psi<\Psi_{1} \\
\boldsymbol{\xi}(\eta) / \sin (\Psi) & \Psi_{1}<\Psi<\pi / 2
\end{array} .\right.
$$

where now $L_{1}(\theta)$ is $L_{1}^{+}$given below Eq. (21).

Thus, for $\eta \rightarrow 1$ the rectangle in Fig. 3 will be long and thin, and thus $\Lambda_{L} \rightarrow$ 0 when $\eta$ is close to one and $\Psi$ is near $\pi / 2$. Thus, as mentioned above, the appearance of the logarithmic singularity is a complicated function of both $\eta$ and $\Psi$, making an entirely numerical approach to the evaluation of the log integrals quite complicated.

In this case the breakpoint in $\Psi, \Psi_{1}$, is a function of $\eta$ and shifting things around will now produce seven integrals instead of the above four. The two starting integrals are

$$
\int_{0}^{1} \mathrm{~d} \eta \int_{0}^{\theta_{\eta}} \mathrm{d} \theta \int_{0}^{\Psi_{1}} \mathrm{~d} \Psi+\int_{0}^{1} \mathrm{~d} \eta \int_{0}^{\theta_{\eta}} \mathrm{d} \theta \int_{\Psi_{1}}^{\pi / 2} \mathrm{~d} \Psi
$$

where $\theta_{\eta}$ is as before, Eq. (22), but now

$$
\Psi_{1}=\Psi_{1}(\eta, \theta)=\tan ^{-1}\left(\boldsymbol{\xi}(\eta) / L_{1}\right)=\tan ^{-1}\left(\frac{1-\eta}{1+\eta}[\sin (\theta)+\sqrt{3} \cos (\theta)]\right)
$$

As in the previous section, the $\theta$ and $\eta$ integrals are easily interchanged, the domain being the region below the $\theta(\eta)$ curve in Fig. 6. This results in the four integrals

$$
\begin{aligned}
\int_{0}^{\pi / 3} \mathrm{~d} \theta \int_{0}^{1} \mathrm{~d} \eta \int_{0}^{\Psi_{1}} \mathrm{~d} \Psi+ & \int_{\pi / 3}^{\pi / 2} \mathrm{~d} \theta \int_{0}^{\eta(\theta)} \mathrm{d} \eta \int_{0}^{\Psi_{1}} \mathrm{~d} \Psi+ \\
& \int_{0}^{\pi / 3} \mathrm{~d} \theta \int_{0}^{1} \mathrm{~d} \eta \int_{\Psi_{1}}^{\pi / 2} \mathrm{~d} \Psi+\int_{\pi / 3}^{\pi / 2} \mathrm{~d} \theta \int_{0}^{\eta(\theta)} \mathrm{d} \eta \int_{\Psi_{1}}^{\pi / 2} \mathrm{~d} \Psi
\end{aligned}
$$

where $\eta(\theta)$ is given in Eq. (36). Using the fact that $\Psi_{1}(\eta=1, \theta)=0$, the geometry for interchanging $\eta$ and $\Psi$ is shown in Fig. 7, $\eta(\theta)<1$, and in Fig. 8, the region below the curve for $\eta(\theta)=1$. Moving the $\eta$ integral to the front in the first two integrals $\left(0<\Psi<\Psi_{1}\right)$ results in

$$
\int_{0}^{\pi / 3} \mathrm{~d} \theta \int_{0}^{\Psi_{00}} \mathrm{~d} \Psi \int_{0}^{\Psi_{\eta}} \mathrm{d} \eta+\int_{\pi / 3}^{\pi / 2} \mathrm{~d} \theta \int_{0}^{\Psi_{\theta}} \mathrm{d} \Psi \int_{0}^{\eta(\theta)} \mathrm{d} \eta+\int_{\pi / 3}^{\pi / 2} \mathrm{~d} \theta \int_{\Psi_{\theta}}^{\Psi_{00}} \mathrm{~d} \Psi \int_{0}^{\Psi_{\eta}} \mathrm{d} \eta
$$




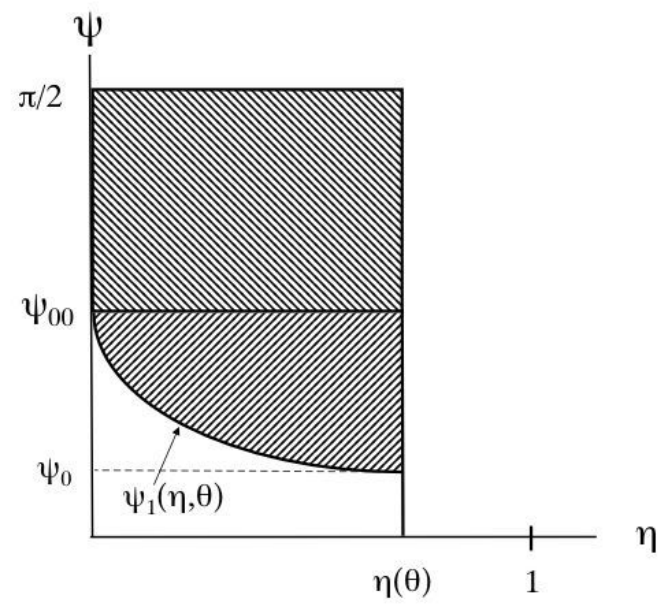

Figure 7: The domain for interchanging the integrals $\{\eta, \Psi\}$, for a fixed value of $\theta$ and $\eta(\theta)<1$.

where

$$
\begin{aligned}
\Psi_{00} & =\Psi_{1}(\eta=0)=\tan ^{-1}([\sin (\theta)+\sqrt{3} \cos (\theta)]) \\
\Psi_{\theta} & =\Psi_{1}(\eta=\eta(\theta), \theta)=\tan ^{-1}\left(\frac{1-\eta(\theta)}{1+\eta(\theta)}[\sin (\theta)+\sqrt{3} \cos (\theta)]\right) \\
\Psi_{\eta} & =\frac{1-\gamma}{1+\gamma} \quad \gamma=\frac{\tan (\Psi)}{\sin (\theta)+\sqrt{3} \cos (\theta)}
\end{aligned}
$$

Similarly, the second two integrals in Eq. (41), $\Psi_{1}<\Psi<\pi / 2$, become

$$
\begin{aligned}
& \int_{0}^{\pi / 3} \mathrm{~d} \theta \int_{0}^{\Psi_{00}} \mathrm{~d} \Psi \int_{\Psi_{\eta}}^{1} \mathrm{~d} \eta+\int_{0}^{\pi / 3} \mathrm{~d} \theta \int_{\Psi_{00}}^{\pi / 2} \mathrm{~d} \Psi \int_{0}^{1} \mathrm{~d} \eta+ \\
& \int_{\pi / 3}^{\pi / 2} \mathrm{~d} \theta \int_{\Psi_{\theta}}^{\Psi_{00}} \mathrm{~d} \Psi \int_{\Psi_{\eta}}^{\eta(\theta)} \mathrm{d} \eta+\int_{\pi / 3}^{\pi / 2} \mathrm{~d} \theta \int_{\Psi_{00}}^{\pi / 2} \mathrm{~d} \Psi \int_{0}^{\eta(\theta)} \mathrm{d} \eta
\end{aligned}
$$




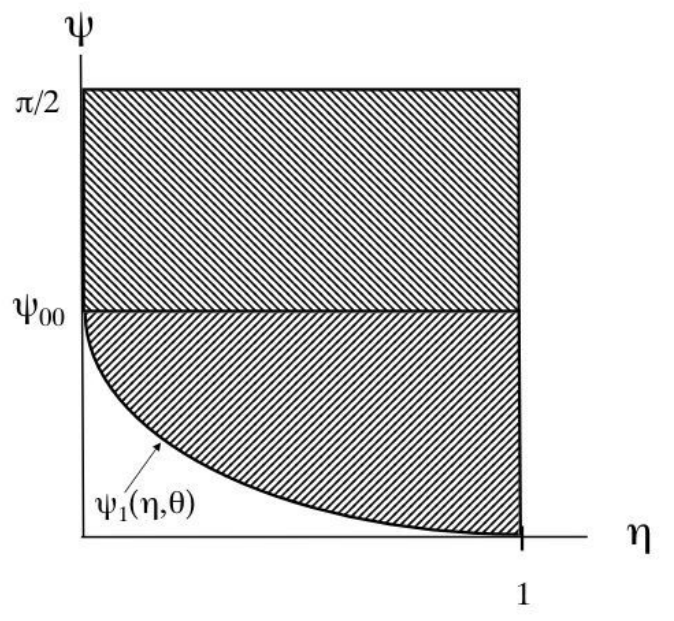

Figure 8: The domain for interchanging the integrals $\{\eta, \Psi\}$, for a fixed value of $\theta, \eta(\theta)=1$.

\section{Test Calculations}

In Galerkin form, the hypersingular equation to be solved is

$$
\int_{\Sigma} \hat{\psi}_{k}(P) \int_{\Sigma}\left(\phi(Q) \frac{\partial^{2} G}{\partial \mathbf{N} \partial \mathbf{n}}(P, Q)-\frac{\partial G}{\partial \mathbf{N}}(P, Q) \frac{\partial \phi}{\partial \mathbf{n}}(Q)\right) \mathrm{d} Q \mathrm{~d} P=0,
$$

it being understood that the the boundary limit is taken exterior to the domain (and thus there is no 'free term' outside of the $Q$ integral). The Galerkin weight functions $\hat{\psi}_{k}(P)$ are comprised of the linear shape functions on the elements. It should be noted that while the methods developed herein have been prompted by the integration of the hypersingular kernel, they are equally applicable to the less singular kernel functions. Thus, analytic integration has also been employed to evaluate the coincident and edge-adjacent integrals involving the first order derivative of $G$.

The numerical evaluation of the integrals, by which we mean Gauss quadrature applied to the original integrals without re-ordering, tends to perform reasonably well for smooth boundaries and regular meshes. In this situation, it is possible that the small errors in the individual integrals (caused by the logarithmic singularity) 
can partially cancel. As evidence of this, we have solved an interior Dirichlet problem on the unit sphere (centered at the origin of the coordinate system), the boundary conditions given by

$$
\phi(x, y, z)=x^{2}+y^{2}-2 z^{2} .
$$

(Note that for Symmetric-Galerkin, Dirichlet problems are solved using the potential equation, not the hypersingular flux equation. However, solving these Dirichlet problems suffice for the testing of the hypersingular evaluation.) This function is harmonic, and thus the exact solution is known, and as it is not linear, the linear element approximation will introduce errors. Denoting the surface area by $|\Sigma|$, the $\mathcal{L}^{2}$ errors are defined as

$$
\left[\frac{1}{|\Sigma|} \int \varepsilon^{2}(Q) \mathrm{d} \Sigma\right]^{1 / 2}
$$

where the error function is $\varepsilon(Q)=f_{c}(Q)-f_{x}(Q), f_{c}$ and $f_{x}$ being the computed and exact values of the flux. For the integration, linear interpolation of the error function is once again employed. Three discretizations of the sphere were employed, 512, 896 and 2048 elements.

Table 1: Integrated $\mathcal{L}^{2}$ errors for the quadratic Dirichlet problem on the unit sphere.

\begin{tabular}{c||ccc|ccc}
\hline \hline \multicolumn{1}{c||}{ Elements } & \multicolumn{3}{c|}{$\eta$ numeric } & \multicolumn{3}{c}{$\eta$ analytic } \\
& $n_{g}=8$ & $n_{g}=12$ & $n_{g}=24$ & $n_{g}=8$ & $n_{g}=12$ & $n_{g}=24$ \\
\hline 512 & 0.05576 & 0.05870 & 0.06063 & 0.06132 & 0.06132 & 0.06132 \\
896 & 0.05853 & 0.03754 & 0.03846 & 0.04002 & 0.03940 & 0.03940 \\
2048 & 0.03793 & 0.02565 & 0.02261 & 0.02300 & 0.02300 & 0.02300 \\
\hline \hline
\end{tabular}

The integrated $\mathcal{L}^{2}$ errors for the sphere problem are shown in Table 1. Although the three meshes are not sufficient to draw any firm conclusions, for the analytic, and numeric with $n_{g}=24$, the convergence is approximately linear in the average element area (defined as $4 \pi$ divided by the number of elements), and hence roughly quadratic in mesh size (the expected behavior). Note that the analytic method has essentially converged by $n_{g}=8$, whereas the numeric may or may not converged with $n_{g}=24$. The slightly better results for the numerical calculation with $n_{g}=24$ is likely due to the fact that this quadrature is employed for the triple integral in the adjacent vertex singular integration [14], while the analytic result again employs $n_{g}=8$. For the two lowest order quadrature levels, the numerical results are clearly somewhat erratic.

The problems in the numerical integration are corroborated by examining the symmetry of the matrix $\mathcal{H}$ generated by the hypersingular integral. The maximum departure from symmetry for a particular row and column $k$ is defined as

$$
\max _{j}\left|\mathcal{H}_{k j}-\mathcal{H}_{j k}\right|
$$


For numerical evaluation, with $n_{g}=24$, on the sphere with 2048 elements, these numbers are on the order $2 \times 10^{-5}$; for the matrix elements, this represents an accuracy of only two to three significant digits. When $\eta$ is integrated analytically, the departure from symmetry is less than $10^{-8}$, and corresponding matrix elements agree to at least 6 digits. Thus, we suspect that the slightly better accuracy for the numerical integration, with $n_{g}=24$, is due to fortuitous cancellation of errors for this smooth geometry. To numerically test the algorithms under more difficult circumstances, we have examined the same Dirichlet problem, only this time employing the unit cube (positive octant) as the domain. This exercises the edge-adjacent evaluation for pairs of elements having a sharp angle, and moreover forces the algorithm to solve for the individual fluxes at edges and corners. As the flux is, in general, discontinuous at these geometric discontinuities, the Galerkin weight functions are chosen to be 'incomplete', i.e., they are non-zero only on one side of the corner/edge [7]; this may inhibit possible cancellation of integration errors at these nodes.

It should be pointed out that, even with these 'incomplete weight functions', the hypersingular integral remains finite: as the potential is continuous, the limit analysis in [14] remains valid and all divergent $\log \left(\epsilon^{2}\right)$ terms cancel. Note too that all individual integrals (coincident, edge) involving the surface flux (one derivative of the Green's function) are finite, and thus the discontinuity of the flux or the choice of weight functions is not a problem for this integral.

Table 2: Integrated $\mathcal{L}^{2}$ errors for the quadratic Dirichlet problem on the unit cube.

\begin{tabular}{c||ccc|ccc}
\hline \hline \multicolumn{1}{c||}{ Elements } & \multicolumn{3}{c|}{$\eta$ numeric } & \multicolumn{3}{c}{$\eta$ analytic } \\
$(h)$ & $n_{g}=8$ & $n_{g}=12$ & $n_{g}=24$ & $n_{g}=8$ & $n_{g}=12$ & $n_{g}=24$ \\
\hline $300(0.200)$ & 0.3615 & 0.3526 & 0.3477 & 0.3461 & 0.3461 & 0.3461 \\
$588(0.1429)$ & 0.2319 & 0.2202 & 0.2139 & 0.2120 & 0.2120 & 0.2120 \\
$972(0.1111)$ & 0.1727 & 0.1570 & 0.1489 & 0.1466 & 0.1465 & 0.1465 \\
$1728(0.0833)$ & 0.1353 & 0.1107 & 0.0988 & 0.0959 & 0.0958 & 0.0958 \\
$3072(0.0625)$ & 0.1258 & 0.0863 & 0.0667 & 0.0626 & 0.0626 & 0.0626 \\
$4332(0.0526)$ & 0.1321 & 0.0809 & 0.0538 & 0.0486 & 0.0485 & 0.0485 \\
$6912(0.0417)$ & 0.1531 & 0.0833 & 0.0422 & 0.0344 & 0.0343 & 0.0343 \\
\hline \hline
\end{tabular}

The cube problem with the quadratic Dirichlet data was solved for various mesh sizes and levels of one-dimensional quadrature; we have employed regular meshes consisting of isosceles right triangle elements with base and height $h$. Based upon the sphere results, we employ a minimum of eight Gauss points for the onedimensional integrations (for the nonsingular integrals, 12-point two-dimensional Gauss rule was used for integrals over the equilateral parameter space). The results, for analytic and numeric evaluation of the coincident and edge-adjacent integrals, are contrasted in Table 2. As expected, due to the presence of corners and edges, the convergence in this case is better than linear, but not quadratic. 
Again examining the symmetry of the $\mathcal{H}$ matrix for the two calculation methods, Table 3 shows the maximum departure from symmetry, Eq. (48), for the row and column corresponding to the corner node $(0,0,0)$. For the analytic calculation there is virtually no change with quadrature level, and thus only the $n_{g}=8$ results are listed. The symmetry using analytic evaluation of the $\eta$ integral is two to three orders of magnitude more accurate than for numerical evaluation, and appropriately decreases with decreasing mesh size. These numbers also demonstrate that the numeric approach has not converged at $n_{g}=24$. Away from corners and edges, both calculations are perfectly symmetric (the non-symmetry on the order of $10^{-12}$ or better), indicating that errors in the numeric integration tend to cancel on a flat surface (note that part of the hypersingular kernel Eq. (4) vanishes if the edge adjacent elements are co-planar).

Table 3: Departure from symmetry at the corner node $(0,0,0)$.

\begin{tabular}{c||ccc|c}
\hline \hline \multicolumn{1}{c||}{ Elements } & \multicolumn{3}{c|}{$\eta$ numeric } & $\eta$ analytic \\
& $n_{g}=8$ & $n_{g}=12$ & $n_{g}=24$ & $n_{g}=8$ \\
\hline 300 & $0.1340 E-03$ & $0.7838 E-04$ & $0.2011 E-04$ & $0.3206 E-07$ \\
588 & $0.1375 E-03$ & $0.6199 E-04$ & $0.1590 E-04$ & $0.2290 E-07$ \\
972 & $0.1147 E-03$ & $0.5170 E-04$ & $0.1327 E-04$ & $0.1781 E-07$ \\
1728 & $0.9262 E-04$ & $0.4177 E-04$ & $0.1072 E-05$ & $0.1336 E-07$ \\
3072 & $0.7443 E-04$ & $0.3357 E-04$ & $0.8616 E-05$ & $0.1002 E-07$ \\
4332 & $0.6517 E-04$ & $0.2940 E-04$ & $0.7546 E-05$ & $0.8437 E-08$ \\
6912 & $0.5428 E-04$ & $0.2449 E-04$ & $0.6286 E-05$ & $0.6680 E-08$ \\
\hline \hline
\end{tabular}

To understand the errors in the cube calculations, two further tests were conducted. First, the discretized (as opposed to integrated) $\mathcal{L}^{2}$ errors

$$
\left[\frac{1}{N} \sum_{j=1}^{N} \varepsilon^{2}\left(n_{j}\right)\right]^{1 / 2}
$$

$\varepsilon\left(n_{j}\right)$ the error at node $n_{j}$ were also computed. The results for the analytic evaluation are given in Table 4 for $n_{g}=8$, higher order quadrature produces the same numbers. If the error function was uniform on the cube surface these numbers would match fairly closely with those in Table 2 . That they do not indicates that the error is not uniform, and indeed this function is significant only at the edges of the cube. The convergence rate is therefore dominated by the slow convergence at the edges, where multiple flux values are computed.

To further check on the origin of this error, the second change was to modify the boundary conditions. Note that there is no error in interpolating the (flat) cube geometry, and the exact flux solution is linear as well. Thus, the only sources of error are from interpolating the quadratic boundary data, Eq. (46), and the integrations (we ignore the small errors from the linear algebra solution). It is 


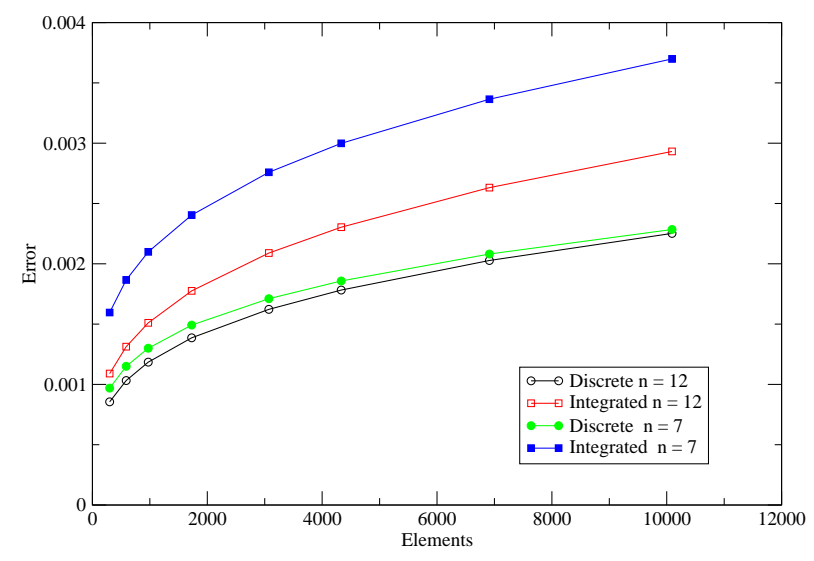

Figure 9: Integrated and discretized errors for the unit cube with linear Dirichlet boundary conditions.

conceivable that the slow convergence at the edges might be due to poor evaluation of the edge-adjacent integral. To demonstrate that this is not the case, the cube calculations were repeated, only this time with linear boundary conditions

$$
\phi(x, y, z)=x+y-2 z .
$$

Figure 9 plots the integrated and discrete $\mathcal{L}^{2}$ errors for the linear boundary condition as a function of the number of elements. The errors are significantly smaller than for the quadratic boundary condition, roughly an order of magnitude, a reflection of the fact that the only remaining source of error is the integration. The increase with decreasing mesh size is a bit surprising at first: however, theorems on convergence assume that integrations are carried out without error, and in this calculation there is only integration error. As the mesh size decreases the area encompassed by the accurate (analytic) singular integrations is reduced, and more of the work is being done by the nonsingular (two-dimensional Gauss quadrature) evaluation. The significant change in results going from $n_{g}=7$ to $n_{g}=12$ for this two-dimensional integration further confirms this as the source of the error.

It can therefore be concluded that the errors at the edges in the quadratic cube calculation stem from the linear interpolation of the boundary data. That this is only pronounced at the edges stems from the fact that, for a smooth surface, the interpolation of the potential on one element says nothing about the flux on its neighboring elements. At an edge however, the approximate potential on one side of the edge is in fact trying to dictate (incorrectly) a directional derivative of $\phi$ on the 'other side' of the edge. This is analogous to the necessary compatability of flux and potential interpolation required for collocating the hypersingular integral at a corner [15]. 
Table 4: Discretzed $\mathcal{L}^{2}$ errors for the quadratic Dirichlet problem on the unit cube.

\begin{tabular}{c||c}
\hline \hline Elements & $n_{g}=8$ \\
\hline 300 & 0.2496 \\
588 & 0.1575 \\
972 & 0.1108 \\
1728 & 0.0737 \\
3072 & 0.0487 \\
4332 & 0.0380 \\
6912 & 0.0271 \\
\hline \hline
\end{tabular}

\subsection{Potential Equation}

As noted earlier, the integration algorithms designed for the hypersingular kernel apply as well to the less singular kernel functions. In this case the integrands that remain after the initial analytic integrations are well behaved, there is no weakly singular logarithm function; nevertheless, these are are by no means simple multidimensional integrals. The benfits of applying the above procedures to the less singular integrals are primarily reduced computational work, and having a consistent treatment of all integrals. Thus, while not absolutely necessary, it is of interest to examine the solutions of the standard boundary integral for surface potential,

$$
\int_{\Sigma} \hat{\Psi}_{k}(P) \int_{\Sigma}\left(\phi(Q) \frac{\partial G}{\partial \mathbf{n}}(P, Q)-G(P, Q) \frac{\partial \phi}{\partial \mathbf{n}}(Q)\right) \mathrm{d} Q \mathrm{~d} P=0
$$

when integrating $\eta$ analytically and numerically.

The unit sphere and cube problems, with quadratic boundary conditions, have therefore been re-solved using the potential equation. Tables 5 and 6 display the corresponding integrated errors. As might be expected, the numerical evaluation does somewhat better for the potential equation, though for the cube, the errors are again somewhat erratic with respect to quadrature level. Despite the good results for $n_{g}=24$ (again likely due to the use of this quadrature level for the adjacent vertex integration), it is not clear whether or not the solutions have converged: the symmetry of the matrix stemming from the Green's function $G(P, Q)$ is much closer to the symmetry in the analytic calculation, but still slightly worse.

\section{Conclusions}

By defining three dimensional Galerkin hypersingular integrals as a boundary limit, their numerical evaluation is reduced to the consideration of finite integrals 
Table 5: Integrated $\mathcal{L}^{2}$ errors for the quadratic Dirichlet problem on the unit sphere, solved using the potential equation..

\begin{tabular}{c||ccc|c}
\hline \hline \multicolumn{1}{c||}{ Elements } & \multicolumn{3}{c|}{$\eta$ numeric } & $\eta$ analytic \\
& $n_{g}=8$ & $n_{g}=12$ & $n_{g}=24$ & $n_{g}=8$ \\
\hline 512 & 0.08663 & 0.07271 & 0.06616 & 0.06435 \\
896 & 0.09092 & 0.06038 & 0.05144 & 0.05104 \\
2048 & 0.05005 & 0.03205 & 0.02481 & 0.02368 \\
\hline \hline
\end{tabular}

Table 6: Integrated $\mathcal{L}^{2}$ errors for the quadratic Dirichlet problem on the unit cube, solved using the potential equation.

\begin{tabular}{c||ccc|c}
\hline \hline Elements & \multicolumn{3}{|c}{$\eta$ numeric } & $\eta$ analytic \\
& $n_{g}=8$ & $n_{g}=12$ & $n_{g}=24$ & $n_{g}=8$ \\
\hline 300 & $0.2993 E+00$ & $0.3187 E+00$ & $0.3405 E+00$ & $0.3495 E+00$ \\
588 & $0.1876 E+00$ & $0.1900 E+00$ & $0.2066 E+00$ & $0.2144 E+00$ \\
972 & $0.1434 E+00$ & $0.1300 E+00$ & $0.1413 E+00$ & $0.1482 E+00$ \\
1728 & $0.1193 E+00$ & $0.8706 E-01$ & $0.9116 E-01$ & $0.9690 E-01$ \\
3072 & $0.1105 E+00$ & $0.6337 E-01$ & $0.5877 E-01$ & $0.6326 E-01$ \\
4332 & $0.1086 E+00$ & $0.5607 E-01$ & $0.4534 E-01$ & $0.4901 E-01$ \\
6912 & $0.1078 E+00$ & $0.5067 E-01$ & $0.3222 E-01$ & $0.3462 E-01$ \\
\hline \hline
\end{tabular}

containing a weak logarithmic singularity. These integrals arise from consideration of the coincident and edge-adjacent singular cases. This paper has demonstrated that, by employing fairly simple re-orderings, the evaluation of these weakly singular integrals can be handled analytically. While more or less essential for treating the hypersingular equation, these methods can also be profitably applied to the standard equation for surface potential.

Extensive testing on a cube problem with Dirichlet boundary conditions has established that the analytic integration procedures work quite well. A benefit of this approach is the reduction in computational effort, the dimension of the coincident numerical integration is reduced from two to one, and for edge-adjacent, from three to two. Moreover, 8 Gauss points is apparently sufficient to achieve a converged solution, much less than what would be required for a completely numeric approach.

As a follow-on to previous work [14], this paper has strictly speaking only dealt with the simplest possible setting, namely the Laplace equation together with a linear element approximation. Nevertheless, the methods are in fact quite general. Most Green's functions can be handled directly, while the extension to higher order interpolations is straightforward, the basic procedure having been discussed in [13]. 


\section{Acknowledgment}

The support of the Spanish Ministry of Education, Culture and Sport through the projects SAB 2003-0088 (LJG), and the Spanish Ministry of Science and Technology through project MAT 2003-03315 (VM), is gratefully acknowledged. AS was supported by the Italian Ministry of University Research (MIUR) under grant EX 60\% - 2005. LJG was also supported by the Applied Mathematical Sciences Research Program of the Office of Mathematical, Information, and Computational Sciences, U.S. Department of Energy, under contract DE-AC05-00OR22725 with UT-Battelle, LLC. LJG would like to thank Prof. Federico Paris and Prof. Angelo Carini for the hospitality at, respectively, the Escuela Superior de Ingenieros, Universidad de Sevilla, and the University of Brescia, where this work was carried out. LJG is also deeply appreciative of numerous fine Italian meals at the home of Mr. and Mrs. Franco Salvadori.

The submitted manuscript has been authored by a contractor of the U. S. Government under contract DE-AC05-00OR22725. Accordingly the U. S. Government retains a non-exclusive, royalty free license to publish or reproduce the published form of this contribution, or allow others to do so, for U. S. Government purposes.

\section{References}

[1] A. Aimi And M. Diligenti, Hypersingular kernel integration in 3D Galerkin boundary element method, J. Comput. Appl. Math., 138 (2002), pp. 51-72.

[2] H. ANDRA, Integration of singular integrals for the Galerkin-type boundary element method in 3D elasticity, Comp. Mech. Appl. Mech. Engng., 157 (1998), pp. 239-249.

[3] M. Bonnet, Boundary Integral Equation Methods for Solids and Fluids, Wiley and Sons, England, 1995.

[4] M. Bonnet and M. Guiggiani, Direct evaluation of double singular integrals and new free terms in $2 D$ (symmetric) Galerkin BEM, Comput. Methods Appl. Mech. Engng., 192 (2003), pp. 2565-2596.

[5] M. Bonnet, G. Maier, And C. Polizzotto, Symmetric Galerkin boundary element method, ASME Appl. Mech. Rev., 51 (1998), pp. 669-704.

[6] B. Danloy, Numerical construction of Gaussian quadrature formulas for $\int_{0}^{1}(-\log x) x^{\alpha} f(x) d x$ and $\int_{0}^{\infty} E_{m}(x) f(x) d x$, Mathematics of Computation, 27 (1973), pp. 861-869. 
[7] F. A. De Paula And J. C. F. Telles, A comparison between point collocation and Galerkin for stiffness matrices obtained by boundary elements, Engineering Analysis with Boundary Elements, 6 (1989), pp. 123-128.

[8] S. Erichsen and S. A. SaUter, Efficient automatic quadrature in 3-D Galerkin BEM, Comp. Mech. Appl. Mech. Engng., 157 (1998), pp. 215-224.

[9] A. Frangi, Regularization of boundary element formulations by the derivative transfer method, in Singular Integrals in the Boundary Element Method, V. Sladek and J. Sladek, eds., Advances in Boundary Elements, Computational Mechanics Publishers, 1998, ch. 4, pp. 125-164.

[10] A. Frangi and M. Guiggiani, Boundary element analysis of Kirchoff plates with direct evaluation of hypersingular integrals, Int. J. Numer. Meth. Engrg., 46 (1999), pp. 1845-1863.

[11] — A direct approach for boundary integral equations with high-order singularities, Int. J. Numer. Meth. Engrg., 49 (2000), pp. 871-898.

[12] A. Frangi, G. Novati, R. Springhetti, and M. Rovizzi, $3 d$ fracture analysis by the symmetric Galerkin BEM, Computational Mechanics, 28 (2002), pp. 220-232.

[13] L. J. GRAY, Evaluation of singular and hypersingular Galerkin boundary integrals: direct limits and symbolic computation, in Singular Integrals in the Boundary Element Method, V. Sladek and J. Sladek, eds., Advances in Boundary Elements, Computational Mechanics Publishers, 1998, ch. 2, pp. 33-84.

[14] L. J. Gray, J. Glaeser, and T. Kaplan, Direct evaluation of hypersingular Galerkin surface integrals, SIAM J. Sci. Comput., 25 (2004), pp. 15341556.

[15] L. J. Gray And E. D. Lutz, On the treatment of corners in the boundary element method, J. Comp. Appl. Math., 32 (1990), pp. 369-386.

[16] L. J. Gray, A.-V. Phan, and T. Kaplan, Boundary integral evaluation of surface derivatives, SIAM J. Sci. Comput., 26 (2004), pp. 294-312.

[17] M. Guiggiani, Formulation and numerical treatment of boundary integral equations with hypersingular kernels, in Singular Integrals in the Boundary Element Method, V. Sladek and J. Sladek, eds., Advances in Boundary Elements, Computational Mechanics Publishers, 1998, ch. 3, pp. 85-124.

[18] N. I. IoAKImidis, A natural approach to the introduction of finite-part integrals into crack problems of three-dimensional elasticity, Engng. Fract. Mech., 16 (1982), pp. 669-673. 
L.J. Gray et al. / Electronic Journal of Boundary Elements, Vol. 4, No. 3, pp. 105-130 (2006)

[19] S. Li, M. E. Mear, AND L. XiaO, Symmetric weak form integral equation method for three-dimensional fracture analysis, Comp. Meth. Appl. Mech. Engng., 151 (1998), pp. 435-459.

[20] P. A. Martin and F. J. Rizzo, On boundary integral equations for crack problems, Proc. R. Soc. Lond., A, 421 (1989), pp. 341-355.

[21] A. Salvadori, Quasi brittle fracture mechanics by cohesive crack models and symmetric Galerkin boundary element method, $\mathrm{PhD}$ thesis, Politecnico Milano, Milan, Italy, 1999.

$[22] \longrightarrow$, Analytical integrations of hypersingular kernel in 3D BEM problems, Comp. Meth. in Appl. Mech. Engng., 190 (2001), pp. 3957-3975.

[23] S. A. Sauter and C. Lage, Transformation of hypersingular integrals and black-box cubature, Mathematics of Computation, 70 (2001), pp. 223-250. 